\title{
Lineare Systemidentifikation des hygrothermischen Verhaltens eines Raumes zur Verwendung in Modellprädiktiven Reglern
}

\author{
Marcel Zehner ${ }^{1}$, Alessio Cavaterra ${ }^{2}$ und Steven Lambeck ${ }^{3}$
}

\section{Zusammenfassung}

Der vorliegende Beitrag beschäftigt sich mit der Linearen Systemidentifikation für ein hygrothermisches Zustandsraummodell des Hrabanus-Maurus-Saals innerhalb der Bibliothek des Bischöflichen Priesterseminars in Fulda. Hier lagern wertvolle historische Dokumente, die besondere Anforderungen an das Raumklima stellen. Für die Einhaltung dieser Anforderungen eignet sich ein Modellprädiktiver Regler (MPC), der in Zukunft im Hrabanus-Maurus-Saal eingesetzt werden wird. Das hier entwickelte lineare Zustandsraummodell kann zukünftig in diesem MPC genutzt werden. Die Modellierung des hygrothermischen Verhaltens erfolgt über eine Beschreibung der physikalischen Zusammenhänge von Temperatur und relativer Luftfeuchtigkeit in Form von Zustandsraummodellen. Die darin enthaltenen Parameter können mit Hilfe der vorhandenen Temperatur - und Luftfeuchtigkeitsmessungen der vergangenen Jahre im Hrabanus-Maurus-Saal geschätzt werden. Hierbei werden eine Vielzahl an Initialparametern in Form einer Monte-Carlo-Simulation getestet, um die optimalen Systemparameter zu identifizieren. Im Anschluss werden beide Zustandsraummodelle zusammengefügt und mit einem rein datengetriebenen Ansatz („Black-Box“-Ansatz) verglichen. Die Ergebnisse des hier vorgestellten Ansatzes („Grey-Box“-Ansatz) weisen vor Allem in Bezug auf die Dynamik ein besseres Modellverhalten auf.

\section{Stichwörter}

Lineare Systemidentifikation, Modellprädiktive Regelung, Präventive Konservierung

\section{Einleitung und Motivation}

Kulturgüter müssen unter bestimmten Bedingungen gelagert oder ausgestellt werden, damit die Haltbarkeit verlängert und deren Wert und Bedeutung künftigen Generationen erhalten bleibt. Bei einer Nicht-Einhaltung dieser Bedingungen kommt es zu einem beschleunigten Alterungsprozess, was mit Beschädigung oder Zerstörung des Gegenstandes verbunden sein kann. Alle Vorkehrungen und Maßnahmen, die getätigt werden müssen, um zu dem dauerhaften Erhalt eines bestimmten Kulturgutes beizutragen, bündeln sich unter dem Begriff der Präventiven Konservierung. Hierbei spielen vor Allem die Temperatur und relative Luftfeuchtigkeit eine besondere Rolle (Burmester, 2007). Dementsprechend ist eine Regelung beider Größen unter Beachtung der vorgegebenen Anforderungen von

Marcel Zehner, B.Eng., Hochschule Fulda, Fachbereich ET

Alessio Cavaterra, M.Eng., Hochschule Fulda, Fachbereich ET

Prof. Dr.-Ing. Steven Lambeck, Hochschule Fulda, Fachbereich ET 
großer Bedeutung. Mit Hilfe eines MPC (Dittmar, 2019) kann eine Mehrgrößenregelung von Temperatur und relativer Luftfeuchtigkeit im Sinne der Präventiven Konservierung erfolgen. Der MPC wird, wie bereits erwähnt, zukünftig im Hrabanus-Maurus-Saal innerhalb der Bibliothek des Bischöflichen Priesterseminars in Fulda eingesetzt. Die dort befindlichen historisch wertvollen Dokumente stellen besondere Anforderungen an das Raumklima. Im Hrabanus-Maurus-Saal finden seit mehreren Jahren durchgehende Temperatur - und Luftfeuchtigkeitsmessungen statt, wodurch auf eine große Datenbasis zurückgegriffen werden kann. Außerdem befinden sich im genannten Raum Stellglieder mit denen Heizeingriffe, aber auch Be - bzw. Entfeuchtungseingriffe durchgeführt werden können. Daneben ist eine Speicherprogrammierbare Steuerung (SPS) vorhanden, die die Mess-, Steuer-, und Regelungstechnik vereint. In Zukunft soll auf dieser SPS auch der MPC implementiert werden. Folgendes Bild 1 zeigt eine Übersicht der bestehenden Sensorik und Aktorik im Hrabanus-Maurus-Saal. An den gekennzeichneten Messstellen werden Temperatur - und Luftfeuchtigkeitsmessungen durchgeführt.

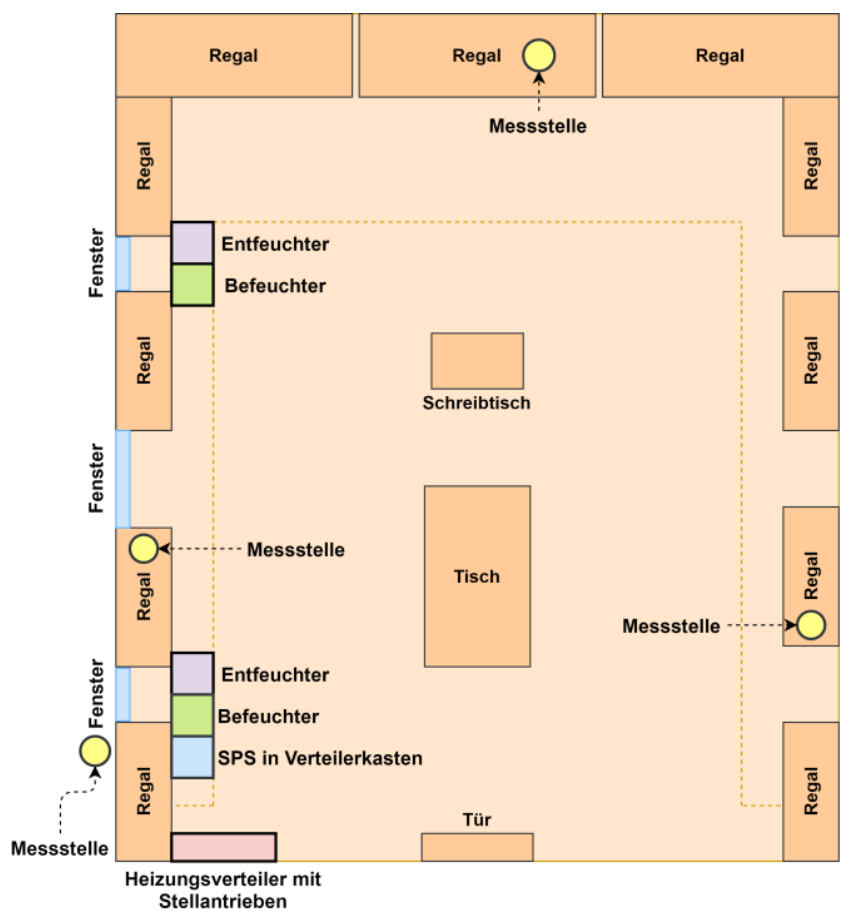

Bild 1: Übersicht über bestehende Sensorik und Aktorik im Hrabanus-Maurus-Saal

Damit der MPC zur Regelung der Temperatur und relativen Luftfeuchtigkeit genutzt werden kann, wird ein hygrothermisches Modell des Hrabanus-Maurus-Saals benötigt. Mit Hilfe des Modells kann der MPC die optimale Stellgrößenfolge unter Berücksichtigung der Stell- und Regelgrößenbeschränkungen gemäß den konservatorischen Anforderungen bestimmen.

\section{Modellbildung und Lineare Systemidentifikation}

Die Modellbildung des hygrothermischen Verhaltens erfolgt mit Hilfe des Beuken-Modells (Cavaterra et al., 2019), wobei das Wärme - und Feuchteübertragungsverhalten durch elektrische Ersatzschaltbilder approximiert wird. Hierzu werden zunächst die Wärme- und Feuchteübertragungsmechanismen in zwei getrennten Zustandsraummodellen zweiter Ordnung abgebildet. Bei der späteren Zusammenführung beider Modelle entsteht ein erweitertes Zustandsraummodell vierter Ordnung für die Temperatur und relative Luftfeuchtigkeit. Das erweiterte Zustandsraummodell besitzt keine Durchgriffsmatrix D. Die Gleichungen (1) und (2) zeigen das Zustandsraummodell zweiter Ordnung für die Temperatur (Wärmeübertragungsverhalten). 


$$
\begin{aligned}
& {\left[\begin{array}{l}
\vartheta_{R} \\
\vartheta_{W}
\end{array}\right]=\left[\begin{array}{cc}
-\frac{1}{C_{R}}\left(\frac{1}{R_{N}}+\frac{1}{R_{\text {fast }}}\right) & \frac{1}{C_{R} R_{W R}} \\
\frac{1}{C_{W} R_{W R}} & -\frac{1}{C_{W}}\left(\frac{1}{R_{A W}}+\frac{1}{R_{W R}}\right)
\end{array}\right]\left[\begin{array}{l}
\vartheta_{R} \\
\vartheta_{W}
\end{array}\right]+\left[\begin{array}{cccc}
\frac{1}{C_{R}} & \frac{\eta_{G, R}}{C_{R}} & \frac{1}{C_{R} R_{\text {fast }}} & \frac{1}{C_{R} R_{N}} \\
0 & \frac{\eta_{G, W}}{C_{W}} & \frac{1}{C_{W} R_{A W}} & 0
\end{array}\right]\left[\begin{array}{c}
Q_{Y} \\
Q_{G} \\
\vartheta_{A} \\
\vartheta_{N}
\end{array}\right]} \\
& y=\left[\begin{array}{ll}
1 & 0
\end{array}\right] \cdot\left[\begin{array}{l}
\vartheta_{R} \\
\vartheta_{W}
\end{array}\right]=\left[\begin{array}{ll}
1 & 0
\end{array}\right] \cdot x_{\vartheta}
\end{aligned}
$$

Unter Berücksichtigung der Außentemperatur $\vartheta_{A}$, der Temperatur des Nachbarraums $\vartheta_{N}$, dem Heizwärmestrom $Q_{Y}$ und dem Wärmestrom der Globalstrahlung $Q_{G}$ kann sowohl die Raumtemperatur $\vartheta_{R}$, als auch die Temperatur an der Wand $\vartheta_{W}$ bestimmt werden. Die vier Eingangsgrößen sind in dem Vektor $u_{\vartheta}$ zusammengefasst. Die Gleichungen (3) und (4) können genutzt werden, um den Wasserdampfpartialdruck zu modellieren und beschreiben somit das Feuchteübertragungsverhalten. Hierbei kann der Wasserdampfpartialdruck im Raum $p_{R}$ und an der Wand $p_{W}$ berechnet werden. Die Eingänge des Systems werden in dem Eingangsvektor $u_{p}$ durch den Wasserdampfpartialdruck außen $p_{A}$, im Nachbarraum $p_{N}$ und dem Wasserdampfmassenstrom $\dot{m}_{Y}$ beschrieben. Die Wasserdampfpartialdrücke eignen sich dazu, dass Feuchteverhalten in Gebäuden zu modellieren. (Kramer et al., 2012)

$$
\begin{gathered}
{\left[\begin{array}{l}
p_{R} \\
p_{W}
\end{array}\right]=\left[\begin{array}{cc}
-\frac{1}{C_{p, R}}\left(\frac{1}{R_{p, N}}+\frac{1}{R_{p, W R}}+\frac{1}{R_{p, \text { fast }}}\right) & \frac{1}{C_{p, R} R_{p, W R}} \\
\frac{1}{C_{p, W} R_{W R}} & -\frac{1}{C_{p, W}}\left(\frac{1}{R_{A W}}+\frac{1}{R_{W R}}\right)
\end{array}\right]\left[\begin{array}{l}
p_{R} \\
p_{W}
\end{array}\right]} \\
+\left[\begin{array}{ccc}
\frac{1}{C_{p, R} R_{p, \text { fast }}} & \frac{1}{C_{p, R} R_{p, N}} & \frac{1}{C_{p, R}} \\
\frac{1}{C_{p, W} R_{p, A W}} & 0 & \frac{1}{C_{p, W}}
\end{array}\right]\left[\begin{array}{l}
p_{A} \\
p_{N} \\
m_{Y}
\end{array}\right] \\
y=\left[\begin{array}{ll}
1 & 0
\end{array}\right] \cdot\left[\begin{array}{l}
p_{R} \\
p_{W}
\end{array}\right]=\left[\begin{array}{ll}
1 & 0
\end{array}\right] \cdot x_{p}
\end{gathered}
$$

Da es sich bei Gleichung (3) um Wasserdampfpartialdrücke handelt, müssen die gemessenen Werte für die relative Luftfeuchtigkeit in Wasserdampfpartialdrücke umgerechnet werden. Erst nach dieser Umrechnung kann die eigentliche Systemidentifikation stattfinden. Mit folgender Berechnungsvorschrift (Kramer et al., 2012) ist dies möglich. Die Funktion $p_{\text {sat }} \vartheta$ ist temperaturabhängig und beschreibt die Wasserdampfpartialdruckkurve (Häupl et al., 2013).

$$
p=p_{\text {sat }} \vartheta \cdot \varphi
$$

Die Parameter innerhalb beider Zustandsraummodelle werden durch die aufgenommenen Messdaten geschätzt. Es erfolgt eine Aufteilung der Messdaten in Trainings - und Testdaten (Aufteilung von 70 \% Trainingsdaten und 30 \% Testdaten). Die Identifikation der Parameter erfolgt mit den Trainingsdaten. Die Evaluierung des daraus entstandenen Modells wird mit den Testdaten durchgeführt. Dies ist notwendig, um das Bias-Variance-Dilemma (Nelles, 2020) zu berücksichtigen. Bild 2 zeigt das Vorgehen zur Ermittlung der Parameter, die anschließend für das hygrothermische Gesamtmodell genutzt werden. Wie in Bild 2 zu erkennen ist, erfolgt zunächst eine Parameterschätzung für die beiden Teilmodelle (Gleichungen (1) und (2) sowie (3) und (4)), bevor eine Zusammenführung zu einem Gesamtmodell mit den ermittelten Systemparametern durchgeführt werden kann. Bei der Zusammenführung erfolgt in Bezug auf das Feuchtemodell eine Linearisierung der Wasserdampfpartialdruckkurve. Die getrennte Identifikation der einzelnen Teilmodelle bewirkt, dass die Schätzaufgabe durch eine geringere Anzahl an Schätzparametern je Teilmodell weniger komplex wird als eine Schätzung der Parameter des Gesamtmodells. Zusätzlich dazu ist die Wahl der entsprechenden Initialparameter von großer Bedeutung. Da bei der Systemidentifikation lokale Optimierungsalgorithmen eingesetzt werden, 
ist es durchaus möglich, dass bei ungünstiger Wahl der Initialparameter ein lokales Minimum erreicht wird und die Konvergenz zum globalen Minimum ausbleibt. Um diesem Sachverhalt entgegenzuwirken, wird mit Hilfe des Latin Hypercube Samplings eine Vielzahl an zufällig verteilten Initialparametervektoren für das Temperatur - und das Feuchtemodell generiert. Diese werden im Anschluss einer Monte-Carlo-Simulation zur Systemidentifikation herangezogen. Das Ergebnis der Monte-Carlo-Simulation ist eine Vielzahl an Modellen mit unterschiedlichen Werten für die Systemparameter. Die Modelle und die dazugehörigen Parameter für Temperatur und Wasserdampfpartialdruck mit dem geringsten Fehler auf den Testdaten werden bei der späteren Zusammenführung zu einem Gesamtmodell genutzt. Als Gütemaß wird die best fit rate (BFR) verwendet. Der normalized mean squared error (NMSE) dient als Fehlermaß. Die Berechnung beider Maße orientiert sich an (Kroll und Schulte, 2014). Die Systemidentifikation selbst erfolgt mittels MATLAB und mit Hilfe der System Identification Toolbox. Unter Verwendung des Befehls greyest kann nach Eingabe der Daten und Festlegung der Zustandsraummodelle die Systemidentifikation stattfinden. Hierfür wird der fmincon-Algorithmus verwendet. Dies kann dem oben beschriebenen Befehl als Parameter übergeben werden.

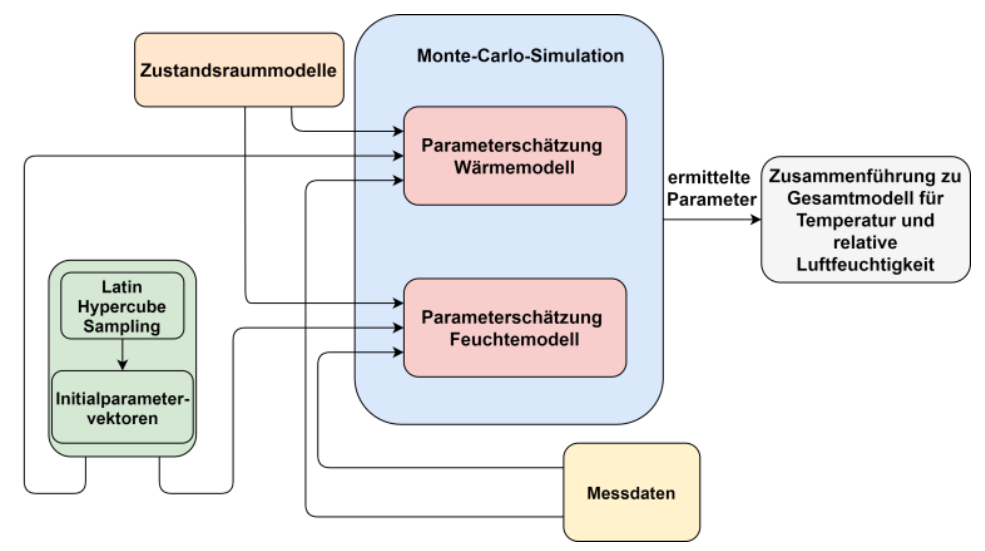

Bild 2: Vorgehen zur Parameterschätzung

Durch die Linearisierung der Wasserdampfpartialkurve (Gleichung (5)), dem anschließenden Einsetzen in das Feuchteübertragungsmodell und dem Zusammenführen beider Modelle ergibt sich ein Zustandsraummodell vierter Ordnung für die Temperatur und relative Luftfeuchtigkeit. Auf die Darstellung der Linearisierung wird im weiteren Verlauf verzichtet, da dies den Rahmen des Beitrags überschreiten würde. Das Gesamtmodell ist in den Gleichungen (8) und (9) zu sehen. Die Systemmatrix $A$ des Gesamtmodells ist nicht vollständig besetzt. Die Elemente $a_{1,3}, a_{1,4}, a_{2,3}$ und $a_{2,4}$ der Matrix sind auf null gesetzt. Dies sagt aus, dass innerhalb des Modells eine Kopplung von der Temperatur auf die relative Luftfeuchtigkeit nicht berücksichtigt wird. Physikalisch betrachtet stellt dies einen Modellierungsfehler dar, der jedoch im vorliegenden Falle nur einen geringen Einfluss auf die Modellierungsgüte hat.

$$
\begin{gathered}
x=\left[\begin{array}{ll}
x_{\vartheta}^{T} & x_{p}^{T}
\end{array}\right]^{T} \\
u=\left[\begin{array}{ll}
u_{\vartheta}^{T} & u_{p}^{T}
\end{array}\right]^{T} \\
x=\boldsymbol{A} \cdot x+\boldsymbol{B} \cdot u \\
y=\left[\begin{array}{llll}
1 & 0 & 0 & 0 \\
0 & 0 & 1 & 0
\end{array}\right] \cdot x
\end{gathered}
$$




\section{Vergleich mit Black-Box-Modell}

Das datengetriebene Modell (Black-Box-Ansatz) ist - wie auch das Grey-Box-Modell - ein Zustandsraummodell vierter Ordnung. Zur besseren Vergleichbarkeit ist die Durchgriffsmatrix D nicht identifiziert worden. Gleichung (10) zeigt die Struktur des Black-Box-Ansatzes mit den Zuständen sowie den Eingangssignalen des Systems. Hierbei stimmen die Dimensionen der Matrizen $\boldsymbol{A}$ und $\boldsymbol{B}$ mit denen aus Gleichung (8) überein. Im Gegensatz zur Systemmatrix $\boldsymbol{A}$ des Grey-Box-Ansatzes werden hier jedoch alle Elemente der Systemmatrix geschätzt.

$$
\left[\begin{array}{l}
\vartheta_{R}(t) \\
\vartheta_{W}(t) \\
\varphi_{R}(t) \\
\varphi_{W}(t)
\end{array}\right]=\boldsymbol{A} \cdot\left[\begin{array}{l}
\vartheta_{R} t \\
\vartheta_{W} t \\
\varphi_{R} t \\
\varphi_{W}
\end{array}\right]+\boldsymbol{B} \cdot\left[\begin{array}{l}
Q_{Y}(t) \\
Q_{G}(t) \\
\vartheta_{A}(t) \\
\vartheta_{N}(t) \\
\varphi_{A}(t) \\
\varphi_{N}(t) \\
m_{Y}(t)
\end{array}\right]
$$

Das Black-Box-Modell und die in den Matrizen enthaltenen Werte sind mit der System Identification Toolbox und dem Befehl ssest identifiziert worden. Dieser Befehl nutzt ebenso den fmincon-Algorithmus. Bild 3 visualisiert den gesamten Testdatensatz, sowie die dazugehörigen Modellausgänge für beide Ansätze. Gleichzeitig ist rechts im Bild ein Bereich zu erkennen, der im weiteren Verlauf genauer untersucht wird. Hierbei handelt es sich um einen Zeitabschnitt, in dem das Grey-Box-Modell schlechter abschneidet im Vergleich zum restlichen Testdatensatz.
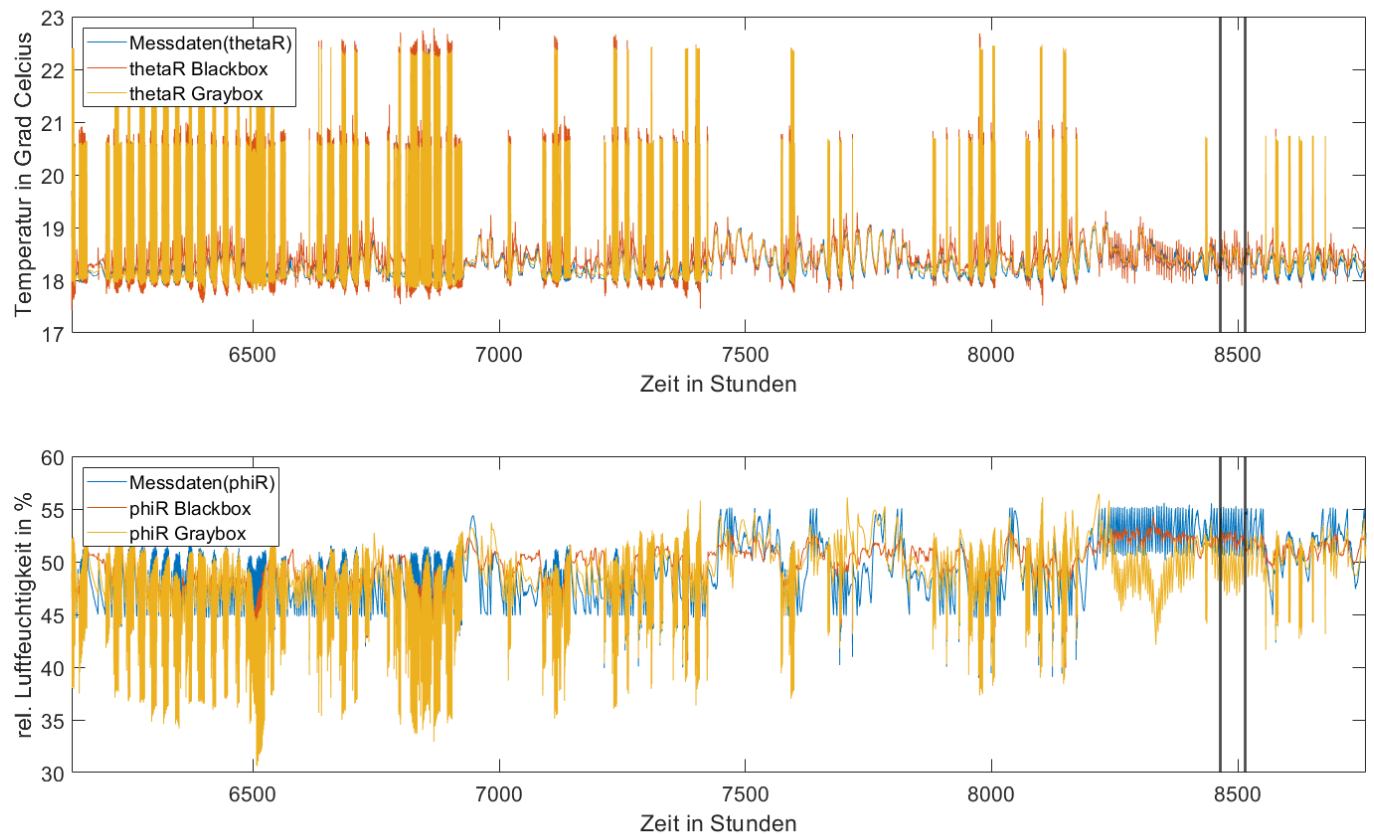

Bild 3: Gesamter Testdatensatz

Der hervorgehobene Bereich in Bild 3 ist in Bild 4 herausgestellt. Auch er veranschaulicht die Messdaten sowie die Ausgänge der beiden Modellierungsansätze. 

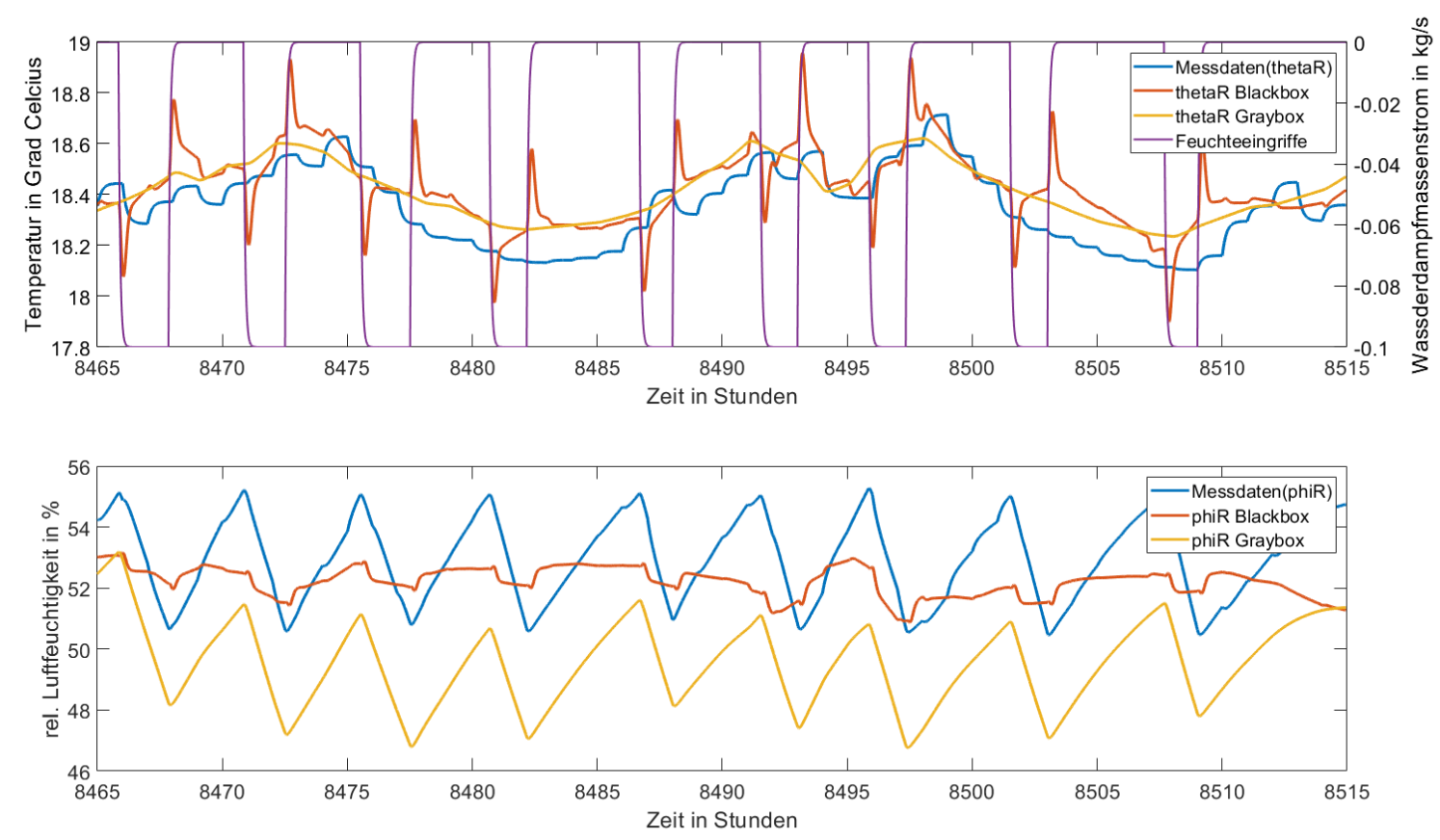

Bild 4: Visualisierung der Modelle, der Messdaten und der Feuchteeingriffe für einen Zeitraum von 50 Stunden

Es wird deutlich, dass der Grey-Box-Ansatz den Verlauf der Temperatur mit hoher Genauigkeit modelliert. Dies zeigt nicht nur Bild 4, sondern auch der berechnete BFR (siehe Tabelle 1). Der Black-BoxAnsatz modelliert ebenfalls den grundsätzlichen Verlauf, jedoch sind hier mehrere Temperaturspitzen zu erkennen. Diese korrelieren mit den Be - bzw. Entfeuchtungseingriffen, die im HrabanusMaurus-Saal vorgenommen wurden und sind in Bild 4 visualisiert. Hier ist erkennbar, dass, bedingt durch zu viele Freiheitsgrade, das rein datengetriebene Modell einen nicht physikalisch messbaren Zusammenhang zwischen Be - bzw. Entfeuchtungseingriffen und der Temperatur gelernt hat. Bei Betrachtung des Grey-Box-Modells für die relative Luftfeuchtigkeit ist erkennbar, dass diese die Dynamik der Messdaten mit hoher Genauigkeit modelliert. Jedoch liegt ein konstanter Offset von ungefähr drei Prozent vor. Dieser Offset ist durch die vorliegende Toleranz der Luftfeuchtigkeitssensoren von ungefähr $\pm 2 \% r H$ jedoch vernachlässigbar. Der Black-Box-Ansatz ist zwar näher an dem Wertebereich der Daten gelegen, was auch die Best Fit Rate in Tabelle 1 zeigt, allerdings kann dieser die Dynamik der Messdaten nicht abbilden. Mit Hilfe des ersten Differenzenquotienten kann nun gezeigt werden, dass mit dem hier vorgestellten Ansatz die Dynamik mit höherer Genauigkeit approximiert werden kann. Dies zeigen nicht nur die berechneten Gütemaße für den Differenzenquotienten in Tabelle 1, sondern auch das Bild 5. Es ist zu erkennen, dass der Grey-Box-Ansatz, die Dynamik der relativen Luftfeuchtigkeit gut modelliert. Lediglich die Spitzen der Messdatenänderung sind von dem hier vorgestellten Ansatz nicht zu erfassen. Im Gegensatz dazu ist der datengetriebene Ansatz (Black-Box) nicht in der Lage die Änderungen der relativen Luftfeuchte darzustellen. 

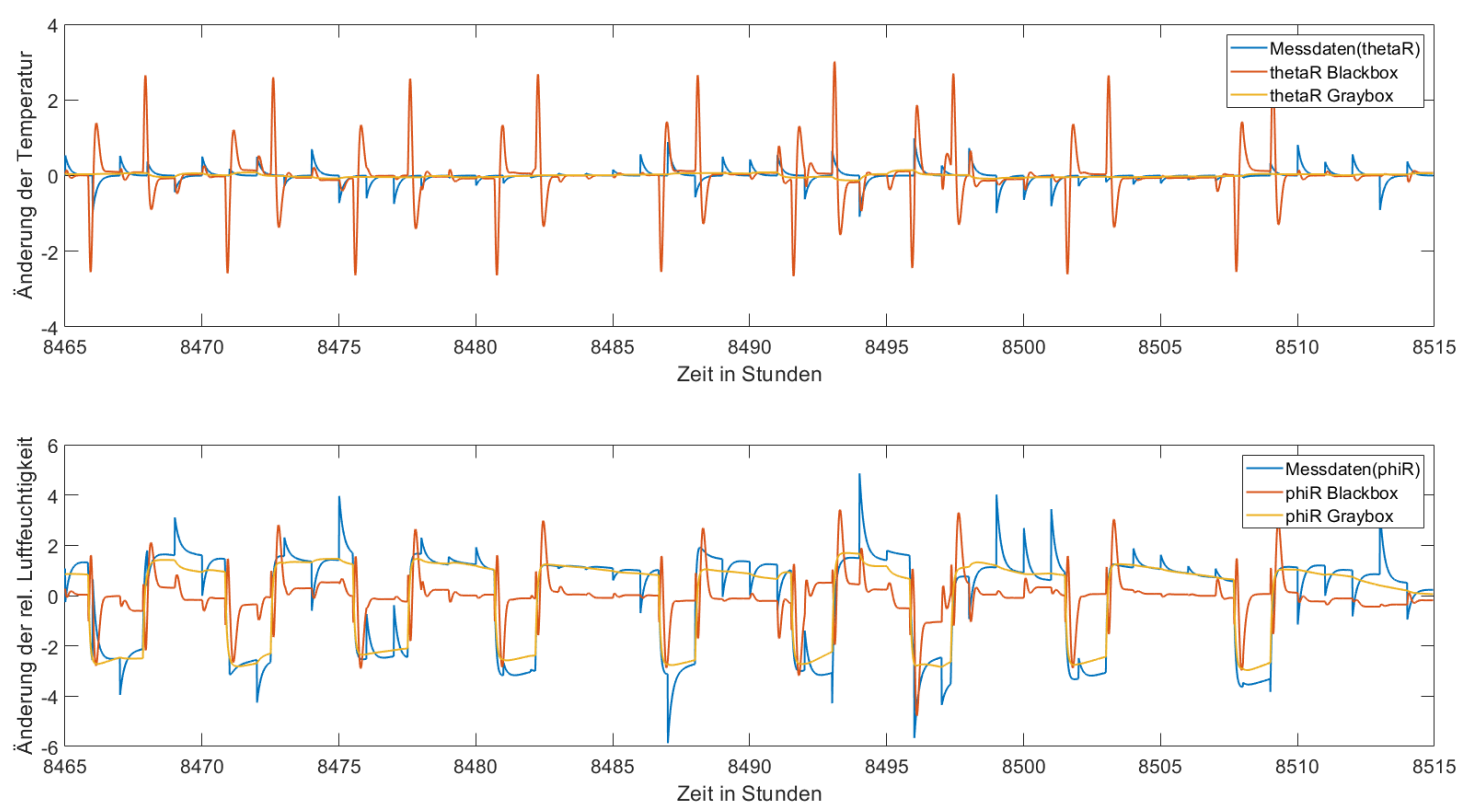

Bild 5: Visualisierung des ersten Differenzenquotienten für den Zeitabschnitt

Die Tatsache, dass der Grey-Box-Ansatz die Dynamik der Luftfeuchtedaten modelliert, jedoch einen konstanten Offset in Bezug auf die Messdaten, kann ebenso mit einem Streudiagramm gezeigt werden. Hierfür werden die in Bild 4 gezeigten Daten in einem Streudiagramm (Bild 6) visualisiert. Auf der Ordinate werden die jeweiligen Modellausgänge gezeigt, wohingegen die Abszisse die Messdaten beinhaltet. Die einzelnen Punktewolken sollten im Idealfall der blau eingezeichneten Winkelhalbierenden folgen und die Steigung dieser abbilden. Ist dies der Fall, so bilden die Modellausgänge die gemessenen Daten exakt ab. Bei Betrachtung der relativen Luftfeuchtigkeit für den Grey-Box-Ansatz ist zu erkennen, dass zwar ein ungefähr dreiprozentiger Offset vorliegt, aber die Punktwolke annähernd
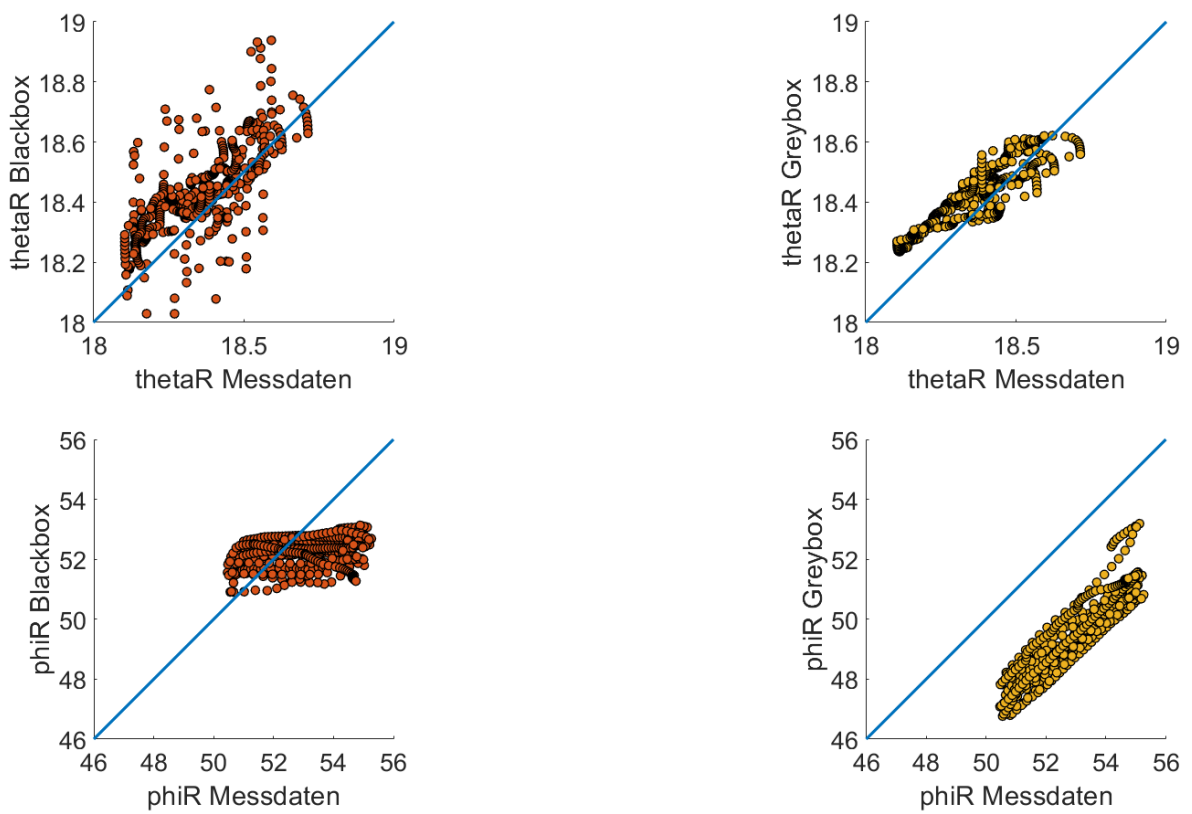

Bild 6: Streudiagramm der Temperatur und relativen Luftfeuchtigkeit 
parallel zur Winkelhalbierenden liegt. Das Streudiagramm für den Black-Box-Ansatz veranschaulicht, dass die Feuchtigkeitswerte von etwas über 53 \%rH nicht abgebildet werden können.

Tabelle 1: Auflistung des BFR und NMSE für den Testdatensatz (DQ = „Differenzenquotient“).

\begin{tabular}{|c|c|c|c|c|}
\hline & BFR & BFR(DQ) & NMSE & NMSE(DQ) \\
\hline Temperatur (Blackbox) & $74,33 \%$ & $77.89 \%$ & 0.066 & 0.049 \\
\hline Relative Luftfeuchtigkeit (Blackbox) & $38,53 \%$ & $75.80 \%$ & 0.38 & 0.059 \\
\hline Temperatur (Greybox) & $90,32 \%$ & $95,06 \%$ & 0.009 & 0,002 \\
\hline Relative Luftfeuchtigkeit (Greybox) & $35,23 \%$ & $80.84 \%$ & 0.42 & 0.037 \\
\hline
\end{tabular}

\section{Fazit und Ausblick}

Der hier vorgestellte Ansatz zeigt eine hohe Modellierungsgenauigkeit bezüglich der Raumtemperatur. Ein Black-Box-Ansatz mit denselben Randbedingungen kann diese Genauigkeit nicht erreichen. Bei Betrachtung der relativen Luftfeuchtigkeit ist jedoch ein größerer Modellfehler vorzufinden, als dies beim datengetriebenen Ansatz der Fall ist. Allerdings zeigt der Verlauf der relativen Luftfeuchtigkeit, dass die Dynamik der Messdaten mit Hilfe des Grey-Box-Ansatzes deutlich besser modelliert werden kann. Diese Aussage wird nochmals durch die Fehlerbetrachtung des ersten Differenzenquotienten für beide Modelle bekräftigt. Der Grey-Box-Ansatz weist bei der Modellierung der relativen Luftfeuchtigkeit einen durchgängig konstanten Offset zu den Messdaten auf, was mit den vorliegenden Toleranzen der Feuchtigkeitssensoren begründet werden kann. In weiterführenden Arbeiten sollte vor Allem der relativen Luftfeuchtigkeit besondere Beachtung geschenkt werden. Hier ist es beispielsweise möglich, eine nichtlineare Systemidentifikation oder eine Takagi-Sugeno-Fuzzy-Modellierung für das Modell durchzuführen, was für zukünftige Arbeiten auch geplant ist.

\section{Literaturverzeichnis}

[1] Alessio Cavaterra, Andreas Böttcher und Steven Lambeck (2019). The "HumFlow" Project - Developing a minimal invasive measurement system for estimating energy and humidity transfer processes through building walls.

[2] Andreas Kroll und Horst Schulte (2014). Benchmark problems for nonlinear system identification and control using Soft Computing methods: Need and overview (25 Ausg.). doi:http://dx.doi.org/10.1016/j.asoc.2014.08.034

[3] Andreas Burmester (2007). Was ist Präventive Konservierung? Eine Einführung. In Grundlagen der Meßtechnik in der Präventiven Konservierung, Tagungsband (S. 8-11). München. Abgerufen am 03.122021 von http://www.doernerinstitut.de/de/mitarbeiter/publikationen_burmester.html

[4] Rainer Dittmar (2019). Model Predictive Control mit MATLAB und Simulink. InTechOpen.

[5] Oliver Nelles (2020). Nonlinear System Identification - From Classical Approaches to NeuralNetworks, Fuzzy Models, and Gaussian Processes. Springer Berlin Heidelberg.

[6] Peter Häupl et al. (2013). Lehrbuch der Bauphysik Schall -- Wärme -- Feuchte -- Licht --Brand --Klima. Springer Vieweg. doi:10.1007/978-3-8348-2101-0

[7] Rick Kramer, Jos van Schijindel und Henk Schellen (2012). Inverse Modeling of climate responses of monumental buildings. In Climate for collections : standards and uncertainties (S. 425-437). 